International Journal of Statistics and Systems

ISSN 0973-2675 Volume 12, Number 4 (2017), pp. 705-719

(C) Research India Publications

http://www.ripublication.com

\title{
Comparative Analysis of Two-Unit Hot Standby Hardware-Software Systems with Impact of Imperfect Fault Coverages
}

\author{
Sudesh Kumari and Rajeev Kumar \\ Department of Mathematics, M.D. University, Rohtak-124001, India.
}

\begin{abstract}
The aim of the paper is to study the impact of imperfect fault coverages on the performance of two-unit hot standby hardware-software systems with the help of stochastic modeling. Two kinds of fault coveragse ie. fault detection and fault recovery coverages are taken here. For the purpose, two stochastic models are developed for the system having two units wherein one unit is operative and other hot standby. In the first model, two-unit hot standby hardware software system with perfect recovery coverage is considered whereas in the second model, possibility of failures in the fault detection and recovery coverages is also included. Using semi-Markov process and regenerative point techniques, various measures of system performance are obtained for the models. The comparative study of the models is carried out to see the impact of imperfect fault coverages in the systems. The comparison of the models is presented with respect to reliability, mean up times, mean degradation times and profit of the system for a particular case. Various conclusions are drawn for the system on the basis of graphical study.
\end{abstract}

Keywords: Two-unit hot standby systems, fault detection coverage, fault recovery coverage, mean up time, mean degradation time, profit, semi-Markov process and regenerative point techniques. 


\section{INTRODUCTION}

In recent years, the systems that continue to functioning properly even on occurrence of fault in some hardware/ software components, i.e fault-tolerant systems have been encouraged. As the reliability of a system get enhanced by avoiding or auto recovery of hardware/ software faults by some inbuilt fault avoiding or fault recovery mechanisms, the fault coverage is an essential key to gain higher reliability in the complex applications. The fault detection coverage is conditional probability of detecting a fault given that fault has occurred whereas the fault recovery coverage is conditional probability of recovery of a fault given that fault has occurred and has been detected. It is noticed that an undetected fault affects the operation of a system and sometimes leads to overall system failure. Also undetected leak, fire or virus infected file may corrupt the system and even lead to a major failure. For assessing reliability of such systems, reliability models are powerful tools.

Different models for a real system covering its different aspects/faults are developed by several researchers including Amari(1), Boyed and Monahan(2), Friedman and Tran(3), Goel et al.(4), Iyer(5), Kanoun and Ortalo-Borrel(6), Kumar and Kumari(8), Rizwan et al.(10), Teng(12), Trivedi et al.(13), Welke et al.(14). All these studies have given attention to the reliability evaluation of hardware/software systems and analyzed single or two unit systems. Some researchers have also given comparison of the models developed for the systems for different situations. For instance, Kumar and Kumar(7), Prashar and Bhardwaj(9), Sharma and Kaur(11) etc. However, there exit many practical situations where hardware-software systems are used with perfect/imperfect recovery coverage.

Keeping this in view, the present paper studies the comparative study of the models carried out to see the impact of imperfect fault coverages in the systems. The comparison of the models is presented with respect to reliability, mean up times, mean degradation times and profit of the system for a particular case. Various conclusions are drawn for the system on the basis of graphical study. For the purpose, two stochastic models are developed for the system having two units wherein one unit is operative and other hot standby. In the first model, two-unit hot standby hardware software system with perfect recovery coverage is considered whereas in the second model, possibility of failures in the fault detection and recovery coverages is also included. In the first model, when operative unit have hardware or software failures, it goes for repair. Then hot standby unit switched into operation, however this lead to degradation of the system. Further on failure of both the units, the system goes to complete failure. The second model accounts for two types of coverages respectively for fault detection and for fault recovery mechanism of the system. Here hardware and software failures are recovered automatically, respectively by hardware coverage and software coverage. In case system is not recovered, the system goes to down state and the infected unit is repaired by the repair facility. Using semi-Markov process and 
regenerative point techniques, various measures of system performance are obtained for the models. Various conclusions are drawn for the systems on the basis of graphical study. Other assumptions are

1. If the system is detected, it is recovered by auto-recovery.

2. In degraded state, the recovery coverage is perfect.

3. If a unit is under repair, it does not work for the system.

4. The time to failures is assumed exponentially distributed whereas other time distributions are general.

5. All random variables are mutually independent.

6. Switching is perfect and instantaneous.

\section{NOTATIONS AND STATES OF THE SYSTEM}

O : Operative unit.

$\mathrm{C}_{\mathrm{d}} / \mathrm{C}_{\mathrm{r}} \quad: \quad$ Fault detection/recovery coverage

$\mathrm{O}_{\mathrm{sC}} / \mathrm{O}_{\mathrm{hC}}: \quad$ Operative unit under coverage due to software/hardware failure

$\mathrm{F}_{\mathrm{sr}} / \mathrm{F}_{\mathrm{hr}}: \quad$ Failed unit under repair on software/hardware failure

$\mathrm{F}_{\mathrm{sR}} / \mathrm{F}_{\mathrm{hR}}$ : $\quad$ Software/Hardware repair is continuing from the previous state.

$\mathrm{F}_{\mathrm{sW}} / \mathrm{F}_{\mathrm{hW}}$ : Failed unit due to software/hardware failure and it is waiting for repair.

$\lambda_{\mathrm{s}} / \lambda_{\mathrm{h}}:$ Software/Hardware failure rate.

$\alpha_{\mathrm{s}} / \alpha_{\mathrm{h}} \quad: \quad$ Software/Hardware repair rate.

$\mathrm{g}_{\mathrm{s}}(\mathrm{t}) / \mathrm{g}_{\mathrm{h}}(\mathrm{t}) \quad$ P.d.f. of time to software/hardware repair.

\section{MODEL-1}

The transition diagram depicting the various states of the system is shown in the fig.1.The epochs of entry into the states $0,1,2$ are regenerative points and thus the states $0,1,2$ are regenerative states and 3, 4, 5, 6 are failed states. Here 1 and 2 states are down states. 


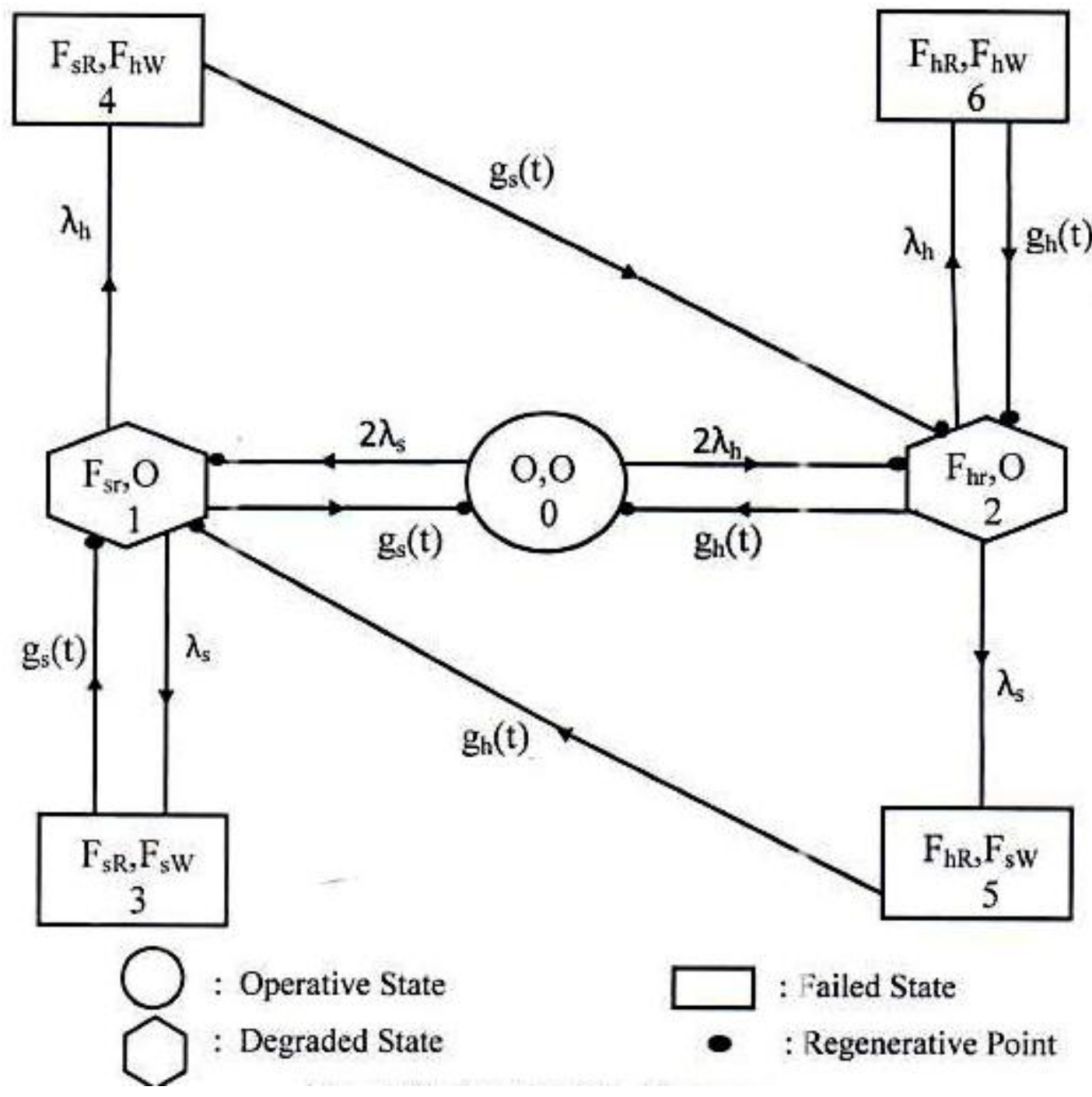

Figure 1: State Transition Diagram

\section{MODEL-II}

The possible transitions of states for the model are shown in the fig.2. The epochs of entry into the states are regenerative points and thus the states $0,1,2,3,4$ are regenerative states. The states $5,6,7,8$ are down states whereas the states $9,10,11$, 12 are failed states. 


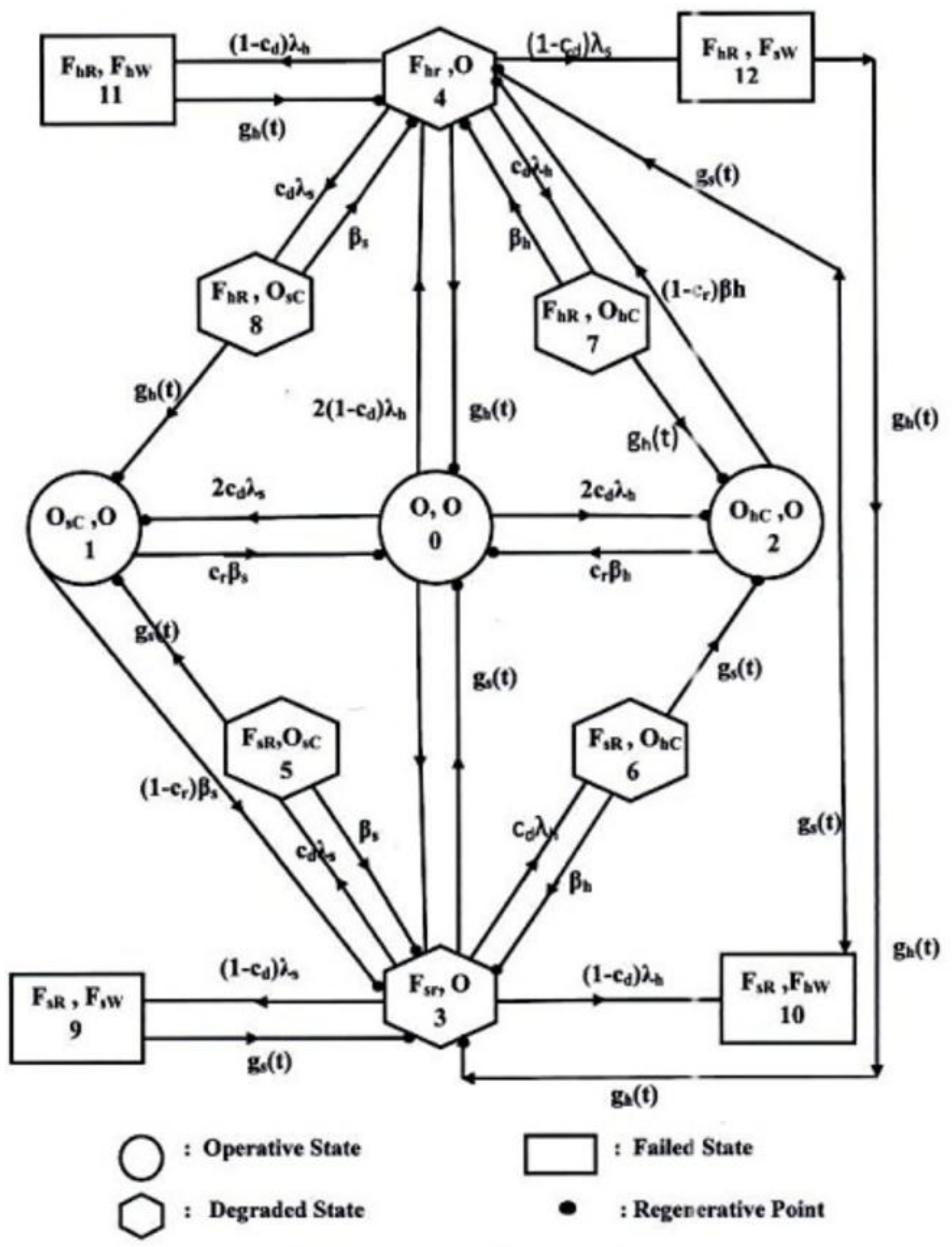

Figure 2: State transition diagram 


\section{MEASURES OF SYSTEM EFFECTIVENESS OF MODEL I}

Using the probabilistic arguments of the theory of regenerative process, various measures of system effectiveness for the model are obtained in steady state:

Mean Time to System Failure $\quad T_{01}=\frac{N_{1}}{D_{1}}$

Mean Up Time of the System $\quad A_{01}=\frac{N_{11}}{D_{11}}$

Mean Degradation Time of the System $\quad D_{01}=\frac{N_{12}}{D_{11}}$

Expected Number of

a) Hardware Repairs

$$
\mathrm{H}_{\mathrm{R} 01}=\frac{\mathrm{N}_{13}}{\mathrm{D}_{11}}
$$

b) Software Repairs

$$
\mathrm{S}_{\mathrm{R} 01}=\frac{\mathrm{N}_{14}}{\mathrm{D}_{11}}
$$

Expected Number of Visits by the Repairman $V_{01}=\frac{N_{15}}{D_{11}}$

where

$$
\begin{aligned}
& \mathrm{D}_{1}=1-\mathrm{p}_{01} \mathrm{p}_{10}-\mathrm{p}_{02} \mathrm{p}_{20} \\
& \mathrm{~N}_{1}=\mu_{0}+\mu_{1} \mathrm{p}_{01}+\mu_{2} \mathrm{p}_{02} \\
& \mathrm{D}_{11}=\mu_{0}\left[\mathrm{p}_{10} \mathrm{p}_{21}^{(5)}+\mathrm{p}_{20}\left(1-\mathrm{p}_{11}^{(3)}\right)\right]+\left(\mathrm{p}_{01} \mathrm{p}_{20}+\mathrm{p}_{21}^{(5)}\right) \mathrm{K}_{1}+\left(1-\mathrm{p}_{01} \mathrm{p}_{10}-\mathrm{p}_{11}^{(3)}\right) \mathrm{K}_{2} \\
& \mathrm{~N}_{11}=\mu_{0}\left[\mathrm{p}_{20}\left(1-\mathrm{p}_{11}^{(3)}\right)+\mathrm{p}_{10} \mathrm{p}_{21}^{(5)}\right] \\
& \mathrm{N}_{12}=\mu_{1}\left(\mathrm{p}_{01} \mathrm{p}_{20}+\mathrm{p}_{21}^{(5)}\right)+\mu_{2}\left(1-\mathrm{p}_{01} \mathrm{p}_{10}-\mathrm{p}_{11}^{(3)}\right) \\
& \mathrm{N}_{13}=\left(1-\mathrm{p}_{01}\right) \mathrm{p}_{10}+\mathrm{p}_{12}^{(4)} \\
& \mathrm{N}_{14}=\mathrm{p}_{01} \mathrm{p}_{20}+\mathrm{p}_{21}^{(5)} \\
& \mathrm{N}_{15}=\left(\mathrm{p}_{10}+\mathrm{p}_{12}^{(4)}\right) \mathrm{p}_{20}+\mathrm{p}_{10} \mathrm{p}_{21}^{(5)}
\end{aligned}
$$

\section{MEASURES OF SYSTEM PERFORMANCE OF MODEL II}

Various measures of system performance obtained in steady state using the arguments of the theory of regenerative process are:
Mean Time to System Failure
$\mathrm{T}_{02}=\frac{\mathrm{N}_{2}}{\mathrm{D}_{2}}$
Mean Up Time of the system
$\mathrm{A}_{02}=\frac{\mathrm{N}_{21}}{\mathrm{D}_{21}}$
Mean Degradation Time of the system

$$
\mathrm{D}_{02}=\frac{\mathrm{N}_{22}}{\mathrm{D}_{21}}
$$




\section{Expected Number of}

a) Hardware Repairs

$$
\mathrm{H}_{\mathrm{R} 02}=\frac{\mathrm{N}_{23}}{\mathrm{D}_{21}}
$$

b) Software Repairs

$$
\mathrm{S}_{\mathrm{R} 02}=\frac{\mathrm{N}_{24}}{\mathrm{D}_{21}}
$$

c) Hardware recovery/detection coverage $H_{C 22}=\frac{N_{25}}{D_{21}}$

d) Software recovery/detection coverage $S_{\mathrm{C} 02}=\frac{N_{26}}{D_{21}}$

\section{Expected Number of Visits by the Repairman $V_{02}=\frac{N_{27}}{D_{21}}$}

where

$$
\begin{aligned}
& \mathrm{D}_{2}=\left[1-\mathrm{p}_{01} \mathrm{p}_{10}-\mathrm{p}_{02} \mathrm{p}_{20}\right]\left[\left\{1-\mathrm{p}_{13} \mathrm{p}_{31}{ }^{(5)}-\mathrm{p}_{33}{ }^{(5)}-\mathrm{p}_{33}{ }^{(6)}\right\}\left\{1-\mathrm{p}_{24} \mathrm{p}_{42}{ }^{(7)}-\mathrm{p}_{44}{ }^{(7)}-\mathrm{p}_{44}{ }^{(8)}\right\}\right. \\
& \left.-\mathrm{p}_{13} \mathrm{p}_{24} \mathrm{p}_{32}{ }^{(6)} \mathrm{p}_{41}{ }^{(8)}\right]-\left[\mathrm{p}_{01} \mathrm{p}_{13}+\mathrm{p}_{03}\right]\left[\left\{\mathrm{p}_{30}+\mathrm{p}_{10} \mathrm{p}_{31}{ }^{(5)}+\mathrm{p}_{20} \mathrm{p}_{32}{ }^{(6)}\right\}\left\{1-\mathrm{p}_{24} \mathrm{p}_{42}{ }^{(7)}-\mathrm{p}_{44}{ }^{(7)}-\mathrm{p}_{44}{ }^{(8)}\right\}\right. \\
& \left.+\mathrm{p}_{24} \mathrm{p}_{32}{ }^{(6)}\left\{\mathrm{p}_{40}+\mathrm{p}_{10} \mathrm{p}_{41}{ }^{(8)}+\mathrm{p}_{20} \mathrm{p}_{42}{ }^{(7)}\right\}\right]-\left[\mathrm{p}_{02} \mathrm{p}_{24}+\mathrm{p}_{04}\right]\left[\mathrm{p}_{13} \mathrm{p}_{41}{ }^{(8)}\left\{\mathrm{p}_{30}+\mathrm{p}_{10} \mathrm{p}_{31}{ }^{(5)}+\mathrm{p}_{20} \mathrm{p}_{32}{ }^{(6)}\right\}\right. \\
& \left.+\left\{\mathrm{p}_{40}+\mathrm{p}_{10} \mathrm{p}_{41}{ }^{(8)}+\mathrm{p}_{20} \mathrm{p}_{42}{ }^{(7)}\right\}\left\{1-\mathrm{p}_{13} \mathrm{p}_{31}{ }^{(5)}-\mathrm{p}_{33}{ }^{(5)}-\mathrm{p}_{33}{ }^{(6)}\right\}\right] \\
& \mathrm{N}_{2}=\mu_{0}\left[\mathrm{p}_{41}{ }^{(8)} \mathrm{p}_{13}\left(\mathrm{p}_{32}{ }^{(6)} \mathrm{p}_{20}-\mathrm{p}_{32}{ }^{(6)}\right)+\left(1-\mathrm{p}_{31}{ }^{(5)} \mathrm{p}_{13}-\mathrm{p}_{33}{ }^{(5)}-\mathrm{p}_{33}{ }^{(6)}\right)\left(1+\mathrm{p}_{42}{ }^{(7)} \mathrm{p}_{20}-\mathrm{p}_{42}{ }^{(7)}-\mathrm{p}_{44}{ }^{(7)}-\mathrm{p}_{44}{ }^{\left({ }^{(8)}\right.}\right)\right] \\
& +\mu_{1}\left[\left(1-\mathrm{p}_{42}{ }^{(7)} \mathrm{p}_{24}-\mathrm{p}_{44}{ }^{(7)}-\mathrm{p}_{44}{ }^{(8)}\right)\left\{\mathrm{p}_{01}\left(1-\mathrm{p}_{33}{ }^{(5)}-\mathrm{p}_{33}{ }^{(6)}\right)+\mathrm{p}_{03} \mathrm{p}_{31}{ }^{(5)}\right\}+\mathrm{p}_{32}{ }^{(6)} \mathrm{p}_{41}{ }^{(8)} \mathrm{p}_{10} \mathrm{p}_{24}\right] \\
& +\mu_{2}\left[\mathrm{p}_{42}{ }^{(7)}\left(1-\mathrm{p}_{01} \mathrm{p}_{10}-\mathrm{p}_{02} \mathrm{p}_{20}\right)\left(1-\mathrm{p}_{33}{ }^{(5)}-\mathrm{p}_{33}{ }^{(6)}-\mathrm{p}_{13} \mathrm{p}_{31}{ }^{(5)}\right)+\mathrm{p}_{32}{ }^{(6)} \mathrm{p}_{41}{ }^{\left({ }^{(8)}\right.} \mathrm{p}_{13}-\mathrm{p}_{42}{ }^{(7)}\left(\mathrm{p}_{01} \mathrm{p}_{13}+\mathrm{p}_{03}\right)\right. \\
& \left(\mathrm{p}_{31}{ }^{(5)} \mathrm{p}_{10}+\mathrm{p}_{32}{ }^{(6)} \mathrm{p}_{20}+\mathrm{p}_{39}+\mathrm{p}_{3,10}\right)-\mathrm{p}_{32}{ }^{(6)}\left(\mathrm{p}_{41}{ }^{(8)} \mathrm{p}_{10}+\mathrm{p}_{42}{ }^{(7)} \mathrm{p}_{20}+\mathrm{p}_{4,11}+\mathrm{p}_{4,12}\right)-\mathrm{p}_{02} \mathrm{p}_{20} \mathrm{p}_{24} \mathrm{p}_{32}{ }^{(6)} \mathrm{p}_{41}{ }^{\left({ }^{(8)}\right.} \\
& \left.+\left(1-\mathrm{p}_{31}{ }^{(5)} \mathrm{p}_{13}-\mathrm{p}_{33}{ }^{(5)}-\mathrm{p}_{33}{ }^{(6)}\right)\left(1+\mathrm{p}_{42}{ }^{(7)} \mathrm{p}_{24}-\mathrm{p}_{42}{ }^{(7)}-\mathrm{p}_{44}{ }^{(7)}-\mathrm{p}_{44}{ }^{(8)}\right)\right]+\mathrm{m}_{3}\left[\left(\mathrm{p}_{01} \mathrm{p}_{13}+\mathrm{p}_{03}\right)\right. \\
& \left.\left(1-\mathrm{p}_{42}{ }^{(7)} \mathrm{p}_{24}-\mathrm{p}_{44}{ }^{(7)}-\mathrm{p}_{44}{ }^{(8)}\right)+\mathrm{p}_{02} \mathrm{p}_{13} \mathrm{p}_{24} \mathrm{p}_{41}{ }^{(8)}\right]+\mathrm{m}_{4}\left[\mathrm{p}_{32}{ }^{(6)} \mathrm{p}_{24}\left(\mathrm{p}_{01} \mathrm{p}_{13}+\mathrm{p}_{03}\right)\right. \\
& \left.+\mathrm{p}_{02} \mathrm{p}_{24}\left(1-\mathrm{p}_{31}{ }^{(5)} \mathrm{p}_{13}-\mathrm{p}_{33}{ }^{(5)}-\mathrm{p}_{33}{ }^{(6)}\right)\right] \\
& \mathrm{D}_{21}=\mu_{0}\left[\left(\mathrm{p}_{30}+\mathrm{p}_{31}{ }^{(5)} \mathrm{p}_{10}+\mathrm{p}_{32}{ }^{(6)} \mathrm{p}_{20}\right)\left(\mathrm{p}_{41}{ }^{(8)} \mathrm{p}_{13}+\mathrm{p}_{43}{ }^{(12)}\right)+\left(1-\mathrm{p}_{31}{ }^{(5)} \mathrm{p}_{13}-\mathrm{p}_{33}{ }^{(5)}-\mathrm{p}_{33}{ }^{(6)}-\mathrm{p}_{33}{ }^{(9)}\right)\right. \\
& \left.\left(\mathrm{p}_{40}+\mathrm{p}_{41}{ }^{(8)} \mathrm{p}_{10}+\mathrm{p}_{42}{ }^{(7)} \mathrm{p}_{20}\right)\right]+\mu_{1}\left[\mathrm{p}_{31}{ }^{(5)}\left(1-\mathrm{p}_{01} \mathrm{p}_{10}-\mathrm{p}_{02} \mathrm{p}_{20}\right)\left(1-\mathrm{p}_{42}{ }^{(7)} \mathrm{p}_{24}-\mathrm{p}_{44}{ }^{(7)}-\mathrm{p}_{44}{ }^{(8)}-\mathrm{p}_{44}{ }^{(11)}\right)\right. \\
& +\mathrm{p}_{41}{ }^{(8)}\left(1-\mathrm{p}_{01} \mathrm{p}_{10}-\mathrm{p}_{02} \mathrm{p}_{20}\right)\left(\mathrm{p}_{32}{ }^{(6)} \mathrm{p}_{24}+\mathrm{p}_{34}{ }^{(10)}\right)+\mathrm{p}_{01}\left(1-\mathrm{p}_{42}{ }^{(7)} \mathrm{p}_{24}-\mathrm{p}_{44}{ }^{(7)}-\mathrm{p}_{44}{ }^{(8)}-\mathrm{p}_{44}{ }^{(11)}\right) \\
& \left(\mathrm{p}_{30}+\mathrm{p}_{31}{ }^{(5)} \mathrm{p}_{10}+\mathrm{p}_{32}{ }^{(6)} \mathrm{p}_{20}\right)+\left(\mathrm{p}_{32}{ }^{(6)} \mathrm{p}_{24}+\mathrm{p}_{34}{ }^{(10)}\right)\left(\mathrm{p}_{40}+\mathrm{p}_{41}{ }^{(8)} \mathrm{p}_{10}+\mathrm{p}_{42}{ }^{(7)} \mathrm{p}_{20}\right) \\
& \left.-\mathrm{p}_{41}{ }^{(8)}\left(\mathrm{p}_{02} \mathrm{p}_{24}+\mathrm{p}_{04}\right)\left(\mathrm{p}_{30}+\mathrm{p}_{31}{ }^{(5)} \mathrm{p}_{10}+\mathrm{p}_{32}{ }^{(6)} \mathrm{p}_{20}\right)-\mathrm{p}_{31}{ }^{(5)}\left(\mathrm{p}_{40}+\mathrm{p}_{41}{ }^{(8)} \mathrm{p}_{10}+\mathrm{p}_{42}{ }^{(7)} \mathrm{p}_{20}\right)\right] \\
& +\mu_{2}\left[p_{42}{ }^{(7)}\left(1-p_{01} p_{10}-p_{02} p_{20}\right)\left(1-p_{31}{ }^{(5)} p_{13}-p_{33}{ }^{(5)}-p_{33}{ }^{(6)}-p_{33}{ }^{(9)}\right)+p_{32}{ }^{(6)}\left(1-p_{01} p_{10}-p_{02} p_{20}\right)\right. \\
& \left(\mathrm{p}_{41}{ }^{(8)} \mathrm{p}_{13}+\mathrm{p}_{43}{ }^{(12)}\right)-\mathrm{p}_{42}{ }^{(7)}\left(\mathrm{p}_{01} \mathrm{p}_{13}+\mathrm{p}_{03}\right)\left(\mathrm{p}_{30}+\mathrm{p}_{31}{ }^{(5)} \mathrm{p}_{10}+\mathrm{p}_{32}{ }^{(6)} \mathrm{p}_{20}\right)+\mathrm{p}_{32}{ }^{(6)}\left(\mathrm{p}_{01} \mathrm{p}_{13}+\mathrm{p}_{03}\right) \\
& \left(\mathrm{p}_{40}+\mathrm{p}_{41}{ }^{(8)} \mathrm{p}_{10}+\mathrm{p}_{42}{ }^{(7)} \mathrm{p}_{20}\right)+\mathrm{p}_{02}\left(\mathrm{p}_{30}+\mathrm{p}_{31}{ }^{(5)} \mathrm{p}_{10}+\mathrm{p}_{32}{ }^{(6)} \mathrm{p}_{20}\right)\left(\mathrm{p}_{41}{ }^{(8)} \mathrm{p}_{13}+\mathrm{p}_{43}{ }^{(12)}\right) \\
& \left.+\left(1-\mathrm{p}_{33}{ }^{(5)}-\mathrm{p}_{33}{ }^{(6)}-\mathrm{p}_{13} \mathrm{p}_{31}{ }^{(5)}\right)\left(\mathrm{p}_{40}+\mathrm{p}_{41}{ }^{(8)} \mathrm{p}_{10}+\mathrm{p}_{42}{ }^{(7)} \mathrm{p}_{20}\right)\right]+\mathrm{k}_{1}\left[\left(1-\mathrm{p}_{01} \mathrm{p}_{10}-\mathrm{p}_{02} \mathrm{p}_{20}\right)\right. \\
& \left.\left(\mathrm{p}_{41}{ }^{(8)} \mathrm{p}_{13}+\mathrm{p}_{43}{ }^{(12)}\right)+\left(\mathrm{p}_{01} \mathrm{p}_{13}+\mathrm{p}_{03}\right)\left(\mathrm{p}_{40}+\mathrm{p}_{41}{ }^{(8)} \mathrm{p}_{10}+\mathrm{p}_{42}{ }^{(7)} \mathrm{p}_{20}\right)\right] \\
& +\mathrm{k}_{2}\left[\left(1-\mathrm{p}_{01} \mathrm{p}_{10}-\mathrm{p}_{02} \mathrm{p}_{20}\right)\left(\mathrm{p}_{32}{ }^{(6)} \mathrm{p}_{24}+\mathrm{p}_{34}{ }^{(10)}\right)+\left(\mathrm{p}_{02} \mathrm{p}_{24}+\mathrm{p}_{04}\right)\left(\mathrm{p}_{30}+\mathrm{p}_{31}{ }^{(5)} \mathrm{p}_{10}+\mathrm{p}_{32}{ }^{(6)} \mathrm{p}_{20}\right)\right]
\end{aligned}
$$




$$
\begin{aligned}
& \mathrm{N}_{21}=\mu_{0}\left[\left(1-\mathrm{p}_{31}{ }^{(5)} \mathrm{p}_{13}-\mathrm{p}_{33}{ }^{(5)}-\mathrm{p}_{33}{ }^{(6)}-\mathrm{p}_{33}{ }^{(9)}\right)\left(1-\mathrm{p}_{42}{ }^{(7)} \mathrm{p}_{24}-\mathrm{p}_{44}{ }^{(7)}-\mathrm{p}_{44}{ }^{\left({ }^{8}\right)}-\mathrm{p}_{44}{ }^{(11)}\right)-\left(\mathrm{p}_{41}{ }^{(8)} \mathrm{p}_{13}+\mathrm{p}_{43}{ }^{(12)}\right)\right. \\
& \left.\left(\mathrm{p}_{32}{ }^{(6)} \mathrm{P}_{24}+\mathrm{p}_{34}{ }^{(10)}\right)\right]+\mu_{1}\left[\mathrm { p } _ { 0 1 } \left\{\left(1-\mathrm{p}_{31}{ }^{(5)} \mathrm{p}_{13}-\mathrm{p}_{33}{ }^{\left({ }^{(5)}\right.}-\mathrm{p}_{33}{ }^{\left({ }^{(6)}\right.}-\mathrm{p}_{33}{ }^{(9)}\right)\left(1-\mathrm{p}_{42}{ }^{(7)} \mathrm{p}_{24}-\mathrm{p}_{44}{ }^{(7)}-\mathrm{p}_{44}{ }^{(8)}-\mathrm{p}_{44}{ }^{(11)}\right)\right.\right. \\
& \left.-\left(\mathrm{p}_{41}{ }^{(8)} \mathrm{p}_{13}+\mathrm{p}_{43}{ }^{(12)}\right)\left(\mathrm{p}_{32}{ }^{(6)} \mathrm{p}_{24}+\mathrm{p}_{34}{ }^{(10)}\right)\right\}+\mathrm{p}_{31}{ }^{(5)}\left(\mathrm{p}_{01} \mathrm{p}_{13}+\mathrm{p}_{03}\right)\left(1-\mathrm{p}_{42}{ }^{(7)} \mathrm{p}_{24}-\mathrm{p}_{44}{ }^{(7)}-\mathrm{p}_{44}{ }^{(8)}-\mathrm{p}_{44}{ }^{(11)}\right) \\
& +\mathrm{p}_{41}{ }^{(8)}\left(\mathrm{p}_{01} \mathrm{p}_{13}+\mathrm{p}_{03}\right)\left(\mathrm{p}_{32}{ }^{(6)} \mathrm{P}_{24}+\mathrm{p}_{34}{ }^{(10)}\right)+\mathrm{p}_{41}{ }^{(8)}\left(\mathrm{p}_{01} \mathrm{p}_{13}+\mathrm{p}_{03}\right)\left(\mathrm{p}_{32}{ }^{(6)} \mathrm{P}_{24}+\mathrm{p}_{34}{ }^{(10)}\right)+\mathrm{p}_{31}{ }^{(5)}\left(\mathrm{p}_{02} \mathrm{p}_{24}+\mathrm{p}_{04}\right) \\
& \left.\left(\mathrm{p}_{41}{ }^{(8)} \mathrm{p}_{13}+\mathrm{p}_{43}{ }^{(12)}\right)+\mathrm{p}_{41}{ }^{(8)}\left(\mathrm{p}_{02} \mathrm{p}_{24}+\mathrm{p}_{04}\right)\left(1-\mathrm{p}_{31}{ }^{(5)} \mathrm{p}_{13}-\mathrm{p}_{33}{ }^{(5)}-\mathrm{p}_{33}{ }^{(6)}-\mathrm{p}_{33}{ }^{(9)}\right)\right] \\
& +\mu_{2}\left[\mathrm { p } _ { 0 2 } \left\{\left(1-\mathrm{p}_{31}{ }^{(5)} \mathrm{p}_{13}-\mathrm{p}_{33}{ }^{(5)}-\mathrm{p}_{33}{ }^{(6)}-\mathrm{p}_{33}{ }^{(9)}\right)\left(1-\mathrm{p}_{42}{ }^{(7)} \mathrm{p}_{24}-\mathrm{p}_{44}{ }^{(7)}-\mathrm{p}_{44}{ }^{(8)}-\mathrm{p}_{44}{ }^{(1)}\right)-\left(\mathrm{p}_{41}{ }^{(8)} \mathrm{p}_{13}+\mathrm{p}_{43}{ }^{(12)}\right)\right.\right. \\
& \left.\left(\mathrm{p}_{32}{ }^{(6)} \mathrm{P}_{24}+\mathrm{p}_{34}{ }^{(10)}\right)\right\}+\mathrm{p}_{32}{ }^{(6)}\left(\mathrm{p}_{01} \mathrm{p}_{13}+\mathrm{p}_{03}\right)\left(1-\mathrm{p}_{42}{ }^{(7)} \mathrm{p}_{24}-\mathrm{p}_{44}{ }^{(7)}-\mathrm{p}_{44}{ }^{(8)}-\mathrm{p}_{44}{ }^{\left({ }^{(1)}\right)}\right)+\mathrm{p}_{42}{ }^{(7)}\left(\mathrm{p}_{01} \mathrm{p}_{13}+\mathrm{p}_{03}\right) \\
& \left(\mathrm{p}_{32}{ }^{(6)} \mathrm{P}_{24}+\mathrm{p}_{34}{ }^{(10)}\right)+\mathrm{p}_{32}{ }^{(6)}\left(\mathrm{p}_{02} \mathrm{p}_{24}+\mathrm{p}_{04}\right)\left(\mathrm{p}_{41}{ }^{(8)} \mathrm{p}_{13}+\mathrm{p}_{43}{ }^{(12)}\right)+\mathrm{p}_{42}{ }^{(7)}\left(\mathrm{p}_{02} \mathrm{p}_{24}+\mathrm{p}_{04}\right) \\
& \left.\left(1-\mathrm{p}_{31}{ }^{(5)} \mathrm{p}_{13}-\mathrm{p}_{33}{ }^{(5)}-\mathrm{p}_{33}{ }^{(6)}-\mathrm{p}_{33}{ }^{(9)}\right)\right] \\
& \mathrm{N}_{22}=\mathrm{p}_{01} \mathrm{p}_{10} \mathrm{p}_{20}\left[\mu_{4} \mathrm{p}_{32}{ }^{(6)}-\mu_{3} \mathrm{p}_{42}{ }^{(7)}\right]+\left[1-\mathrm{p}_{02} \mathrm{p}_{20}\right]\left[\mathrm{p}_{10}\left\{\mu_{4} \mathrm{p}_{31}{ }^{(5)}-\mu_{3} \mathrm{p}_{41}{ }^{(8)}\right\}-\mu_{3} \mathrm{p}_{40}\right] \\
& \mathrm{N}_{23}=\left[1-\mathrm{p}_{44}{ }^{(7)}-\mathrm{p}_{44}{ }^{\left({ }^{())}\right.}\right]\left[\mathrm{p}_{10} \mathrm{p}_{31}{ }^{(5)}\left(1-\mathrm{p}_{02} \mathrm{p}_{20}\right)-\mathrm{p}_{01} \mathrm{p}_{10} \mathrm{p}_{24} \mathrm{p}_{32}{ }^{(6)}\right] \\
& \mathrm{N}_{24}=\left[1-\mathrm{p}_{33}{ }^{\left({ }^{()}\right.}-\mathrm{p}_{33}{ }^{\left({ }^{6}\right)}\right]\left[\begin{array}{l}
\left(1-\mathrm{p}_{02} \mathrm{p}_{20}\right)\left(\mathrm{p}_{13} \mathrm{p}_{41}{ }^{(8)}-\mathrm{p}_{40}-\mathrm{p}_{41}{ }^{(8)}-\mathrm{p}_{44}{ }^{(7)}-\mathrm{p}_{44}{ }^{(8)}\right) \\
-\mathrm{p}_{01} \mathrm{p}_{10}\left(1-\mathrm{p}_{24} \mathrm{p}_{42}{ }^{(7)}-\mathrm{p}_{44}{ }^{(1)}\right)
\end{array}\right] \\
& \mathrm{N}_{25}=\mathrm{p}_{02} \mathrm{p}_{20}\left[\left\{1-\mathrm{p}_{13} \mathrm{p}_{31}{ }^{(5)}-\mathrm{p}_{33}{ }^{(5)}-\mathrm{p}_{33}{ }^{(6)}-\mathrm{p}_{33}{ }^{(9)}\right\}\left\{1-\mathrm{p}_{24} \mathrm{p}_{42}{ }^{(7)}-\mathrm{p}_{44}{ }^{(7)}-\mathrm{p}_{44}{ }^{(8)}-\mathrm{p}_{44}{ }^{(11)}\right\}\right. \\
& \left.-\left\{\mathrm{p}_{13} \mathrm{p}_{41}{ }^{(8)}+\mathrm{p}_{43}{ }^{(12)}\right\}\left\{\mathrm{p}_{24} \mathrm{p}_{32}{ }^{(6)}+\mathrm{p}_{34}{ }^{(10)}\right\}\right]+\left[\mathrm{p}_{20} \mathrm{p}_{32}{ }^{(6)}+\mathrm{p}_{33}{ }^{(6)}\right]\left[\left\{\mathrm{p}_{01} \mathrm{p}_{13}+\mathrm{p}_{03}\right\}\right. \\
& \left.\left\{1-\mathrm{p}_{24} \mathrm{p}_{42}{ }^{(7)}-\mathrm{p}_{44}{ }^{(7)}-\mathrm{p}_{44}{ }^{(8)}-\mathrm{p}_{44}{ }^{(11)}\right\}+\left\{\mathrm{p}_{02} \mathrm{p}_{24}+\mathrm{p}_{04}\right\}\left\{\mathrm{p}_{13} \mathrm{p}_{41}{ }^{(8)}+\mathrm{p}_{43}{ }^{(12)}\right\}\right]+\left[\mathrm{p}_{20} \mathrm{p}_{42}{ }^{(7)}+\mathrm{p}_{44}{ }^{(7)}\right] \\
& {\left[\left\{\mathrm{p}_{01} \mathrm{p}_{13}+\mathrm{p}_{03}\right\}\left\{\mathrm{p}_{24} \mathrm{p}_{32}{ }^{(6)}+\mathrm{p}_{34}{ }^{(10)}\right\}+\left\{\mathrm{p}_{02} \mathrm{p}_{24}+\mathrm{p}_{04}\right\}\left\{1-\mathrm{p}_{13} \mathrm{p}_{31}{ }^{(5)}-\mathrm{p}_{33}{ }^{(5)}-\mathrm{p}_{33}{ }^{(6)}-\mathrm{p}_{33}{ }^{(9)}\right\}\right]} \\
& \mathrm{N}_{26}=\mathrm{p}_{01} \mathrm{p}_{10}\left[\left\{1-\mathrm{p}_{13} \mathrm{p}_{31}{ }^{(5)}-\mathrm{p}_{33}{ }^{(5)}-\mathrm{p}_{33}{ }^{(6)}-\mathrm{p}_{33}{ }^{(9)}\right\}\left\{1-\mathrm{p}_{24} \mathrm{p}_{42}{ }^{(7)}-\mathrm{p}_{44}{ }^{(7)}-\mathrm{p}_{44}{ }^{(8)}-\mathrm{p}_{44}{ }^{(11)}\right\}\right. \\
& \left.-\left\{\mathrm{p}_{13} \mathrm{p}_{41}{ }^{(8)}+\mathrm{p}_{43}{ }^{(12)}\right\}\left\{\mathrm{p}_{24} \mathrm{p}_{32}{ }^{(6)}+\mathrm{p}_{34}{ }^{(10)}\right\}\right]+\left[\mathrm{p}_{10} \mathrm{p}_{31}{ }^{(5)}+\mathrm{p}_{33}{ }^{(5)}\right]\left[\left\{\mathrm{p}_{01} \mathrm{p}_{13}+\mathrm{p}_{03}\right\}\right. \\
& \left.\left\{1-\mathrm{p}_{24} \mathrm{p}_{42}{ }^{(7)}-\mathrm{p}_{44}{ }^{(7)}-\mathrm{p}_{44}{ }^{(8)}-\mathrm{p}_{44}{ }^{(11)}\right\}+\left\{\mathrm{p}_{02} \mathrm{p}_{24}+\mathrm{p}_{04}\right\}\left\{\mathrm{p}_{13} \mathrm{p}_{41}{ }^{(8)}+\mathrm{p}_{43}{ }^{(12)}\right\}\right] \\
& +\left[\mathrm{p}_{10} \mathrm{p}_{41}{ }^{(8)}+\mathrm{p}_{44}{ }^{(8)}\right]\left[\begin{array}{l}
\left\{\mathrm{p}_{01} \mathrm{p}_{13}+\mathrm{p}_{03}\right\}\left\{\mathrm{p}_{24} \mathrm{p}_{32}{ }^{(6)}+\mathrm{p}_{34}{ }^{(10)}\right\}+\left\{\mathrm{p}_{02} \mathrm{p}_{24}+\mathrm{p}_{04}\right\} \\
\left\{1-\mathrm{p}_{13} \mathrm{p}_{31}{ }^{(5)}-\mathrm{p}_{33}{ }^{(5)}-\mathrm{p}_{33}{ }^{(6)}-\mathrm{p}_{33}{ }^{(9)}\right\}
\end{array}\right] \\
& \mathrm{N}_{27}=\left[1-\mathrm{p}_{01} \mathrm{p}_{10}-\mathrm{p}_{02} \mathrm{p}_{20}\right]\left[\left\{1-\mathrm{p}_{13} \mathrm{p}_{31}{ }^{(5)}-\mathrm{p}_{33}{ }^{(5)}-\mathrm{p}_{33}{ }^{(6)}-\mathrm{p}_{33}{ }^{(9)}\right\}\right. \\
& \left.\left\{1-\mathrm{p}_{24} \mathrm{p}_{42}{ }^{(7)}-\mathrm{p}_{44}{ }^{(7)}-\mathrm{p}_{44}{ }^{(8)}-\mathrm{p}_{44}{ }^{(1)}\right\}-\left\{\mathrm{p}_{13} \mathrm{p}_{41}{ }^{(8)}+\mathrm{p}_{43}{ }^{(12)}\right\}\left\{\mathrm{p}_{24} \mathrm{p}_{32}{ }^{(6)}+\mathrm{p}_{34}{ }^{(10)}\right\}\right] \\
& +\left[\mathrm{p}_{01} \mathrm{p}_{13}+\mathrm{p}_{03}\right]\left[\left\{\mathrm{p}_{13} \mathrm{p}_{31}{ }^{(5)}+\mathrm{p}_{24} \mathrm{p}_{32}{ }^{(6)}\right\}\left\{1-\mathrm{p}_{24} \mathrm{p}_{42}{ }^{(7)}-\mathrm{p}_{44}{ }^{(7)}-\mathrm{p}_{44}{ }^{(8)}-\mathrm{p}_{44}{ }^{(11)}\right\}\right. \\
& \left.+\left\{\mathrm{p}_{24} \mathrm{p}_{32}{ }^{(6)}+\mathrm{p}_{34}{ }^{(10)}\right\}\left\{\mathrm{p}_{13} \mathrm{p}_{41}{ }^{(8)}+\mathrm{p}_{24} \mathrm{p}_{42}{ }^{(7)}\right\}\right]+\left\{\mathrm{p}_{02} \mathrm{p}_{24}+\mathrm{p}_{04}\right\} \\
& {\left[\begin{array}{l}
\left\{\mathrm{p}_{13} \mathrm{p}_{31}{ }^{(5)}+\mathrm{p}_{24} \mathrm{p}_{32}{ }^{(6)}\right\}\left\{\mathrm{p}_{13} \mathrm{p}_{41}{ }^{(8)}+\mathrm{p}_{43}{ }^{(12)}\right\}+\left\{\mathrm{p}_{13} \mathrm{p}_{41}{ }^{(8)}+\mathrm{p}_{24} \mathrm{p}_{42}{ }^{(7)}\right\} \\
\left\{1-\mathrm{p}_{13} \mathrm{p}_{31}{ }^{(5)}-\mathrm{p}_{33}{ }^{(5)}-\mathrm{p}_{33}{ }^{(6)}-\mathrm{p}_{33}{ }^{(9)}\right\}
\end{array}\right]}
\end{aligned}
$$




\section{PROFIT ANALYSIS OF MODEL I}

The expected total profit $\left(\mathrm{P}_{0}\right)$ incurred to the system in steady state is given by $\mathrm{P}_{01}=\mathrm{C}_{0} \mathrm{~A}_{01}+\mathrm{C}_{1} \mathrm{D}_{01}-\mathrm{C}_{2} \mathrm{H}_{\mathrm{R} 01}-\mathrm{C}_{3} \mathrm{~S}_{\mathrm{R} 01}-\mathrm{C}_{4} \mathrm{~V}_{01}-\mathrm{C}_{5}$

where

$\mathrm{C}_{0}=$ revenue per unit up time of the system.

$\mathrm{C}_{1}=$ revenue per unit degradation time of the system.

$\mathrm{C}_{2}=$ cost per unit of hardware repair.

$\mathrm{C}_{3}=$ cost per unit of software repair.

$\mathrm{C}_{4}=$ cost per visit of the repairman.

$\mathrm{C}_{5}=$ cost per unit of installation.

\section{PROFIT ANALYSIS OF MODEL II}

The expected total profit $\left(\mathrm{P}_{0}\right)$ incurred to the system in steady state is given by $\mathrm{P}_{02}=\mathrm{C}_{0} \mathrm{~A}_{02}+\mathrm{C}_{1} \mathrm{D}_{02}-\mathrm{C}_{2} \mathrm{H}_{\mathrm{R} 02}-\mathrm{C}_{3} \mathrm{~S}_{\mathrm{R} 02}-\mathrm{C}_{4} \mathrm{H}_{\mathrm{C} 02}-\mathrm{C}_{5} \mathrm{~S}_{\mathrm{C} 02}-\mathrm{C}_{6} \mathrm{~V}_{02}-\mathrm{C}_{7}$

where

$\mathrm{C}_{0}=$ revenue per unit up time of the system.

$\mathrm{C}_{1}=$ revenue per unit degradation time of the system.

$\mathrm{C}_{2}=$ cost per unit of hardware repair.

$\mathrm{C}_{3}=$ cost per unit of software repair.

$\mathrm{C}_{4}=$ cost per unit of hardware coverage.

$\mathrm{C}_{5}=$ cost per unit of software coverage.

$\mathrm{C}_{6}=$ cost per visit of the repairman.

$\mathrm{C}_{7}=$ installation cost per unit

\section{PARTICULAR CASE}

The following particular case is considered for the model:

$$
\mathrm{g}_{\mathrm{s}}(\mathrm{t})=\alpha_{\mathrm{s}} \mathrm{e}^{-\alpha_{\mathrm{s}} \mathrm{t}} ; \quad \mathrm{g}_{\mathrm{h}}(\mathrm{t})=\alpha_{\mathrm{h}} \mathrm{e}^{-\alpha_{\mathrm{h}} \mathrm{t}}
$$

where $\alpha_{\mathrm{s}}$ and $\alpha_{\mathrm{h}}$ aresoftwareand hardware repair rates respectively

The assumed values of parameters are as:

software failure rate $\left(\lambda_{s}\right)=0.002$, hardware failure rate $\left(\lambda_{h}\right)=0.001$, software repair rate $\left(\alpha_{\mathrm{s}}\right)=0.9$, hardware repair rate $\left(\alpha_{\mathrm{h}}\right)=1.8$, software recovery rate $\left(\beta_{\mathrm{s}}\right)=1.0$, hardware recovery rate $\left(\beta_{\mathrm{h}}\right)=2.0$, fault detection coverage $\left(\mathrm{C}_{\mathrm{d}}\right)=0.8$, fault recovery coverage $\left(\mathrm{C}_{\mathrm{r}}\right)=0.9, \mathrm{C}_{0}=30,000, \mathrm{C}_{1}=25,000, \mathrm{C}_{2}=2,000, \mathrm{C}_{3}=15,00 \mathrm{C}_{4}=1,000, \mathrm{C}_{5}=$ $900, \mathrm{C}_{6}=200, \mathrm{C}_{7}=150$,

\section{COMPARATIVE ANALYSIS BETWEEN MODEL I AND MODEL II}

Various graphs have been plotted for the Mean time to system failure, Mean up time 
of the system and profit incurred for the system by taking the assumed values of hardware/software failure rate(s), fault detection coverage, fault recovery coverage and hardware/software repair rate(s). Following interpretations have been made from the graphs.

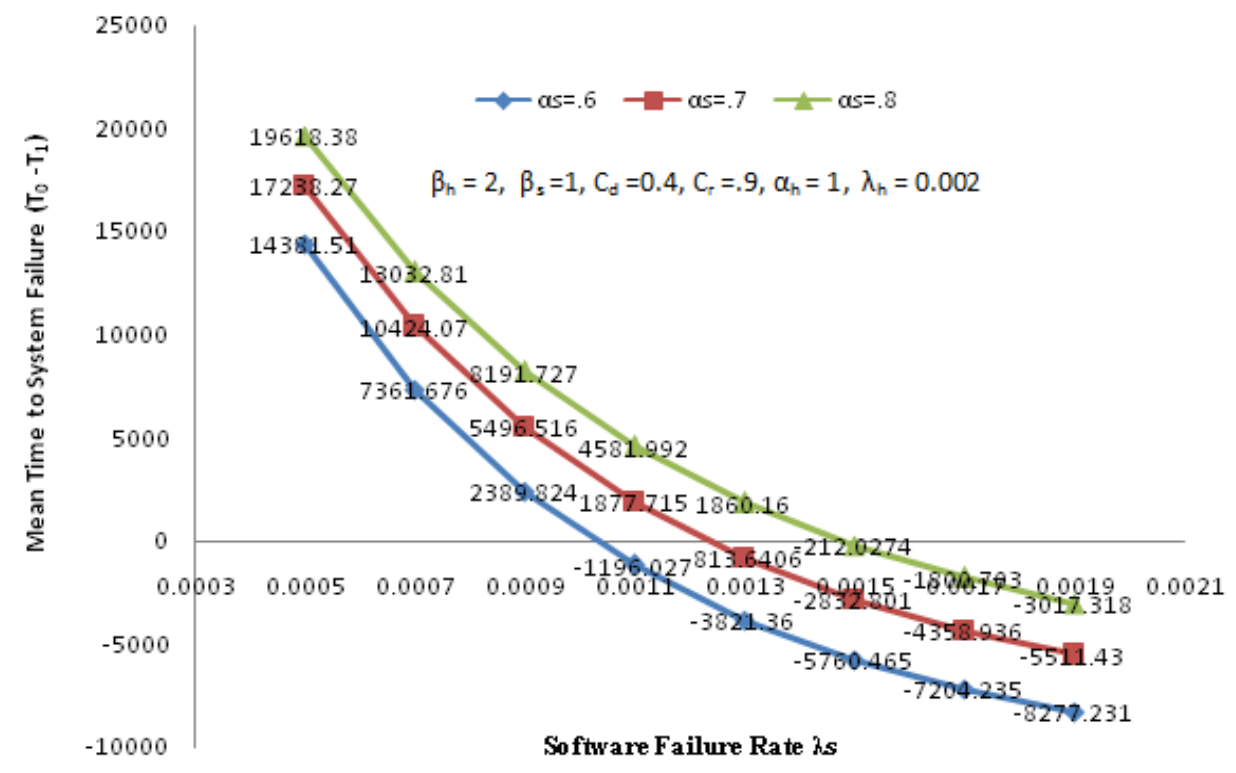

Fig. 3. Mean Time to System Failure versus software failure rate for different values of software repair rate

Fig. 3 depicts the behavior of difference of mean times to system failure

( $\left.\mathbf{T}_{01}-\mathbf{T}_{02}\right)$ of model I and model II with respect to software failure rate $\left(\lambda_{s}\right)$. It can be observed that difference of mean times to system failure $\left(\mathbf{T}_{\mathbf{0 1}}-\mathbf{T}_{\mathbf{0 2}}\right)$ decreases as failure rate increase and has higher values for higher values of software repair rate $\left(\alpha_{\mathrm{s}}\right)$. So, it can be interpreted from the graph that mean time to system failure $\left(\mathrm{T}_{01}\right)$ of model $\mathrm{I}$ is greater than the mean time to system failure $\left(\mathrm{T}_{02}\right)$ of model II for all increasing values of failure rate and repair rate. So, model $\mathrm{I}$ is better in terms of reliability of the system. For $\alpha_{\mathrm{s}}=0.6$, the difference ( $\left.\mathbf{T}_{\mathbf{0 1}}-\mathbf{T}_{\mathbf{0 2}}\right)$ is positive or negative according as $\lambda_{\mathrm{s}}<$ or $>0.00103$. Therefore, the model I is better or no better than the model II whenever $\lambda_{\mathrm{s}}<$ or $>0.00103$. When $\lambda_{\mathrm{s}}=0.00103$, both the models are equally good. For $\alpha_{\mathrm{s}}=0.7$, the difference ( $\left.\mathbf{T}_{\mathbf{0 1}}-\mathbf{T}_{\mathbf{0 2}}\right)$ is positive or negative according as $\lambda_{\mathrm{s}}<$ or $>0.00124$. Therefore, the model I is better or no better than the model II whenever $\lambda_{s}<$ or $>0.00124$. When $\lambda_{\mathrm{s}}=0.00124$, both the models are equally good. For $\alpha_{\mathrm{s}}=0.8$, the difference $\left(\mathbf{T}_{\mathbf{0 1}}-\mathbf{T}_{\mathbf{0 2}}\right)$ is positive or negative according as $\lambda_{\mathrm{s}}<$ or $=$ or $>0.00148$. Therefore, the model $\mathrm{I}$ is better or worse than the model II whenever $\lambda_{\mathrm{s}}<$ or $>0.00148$. When $\lambda_{\mathrm{s}}=0.00148$, both the models are equally good. Therefore, suggestion is given to the user of the system 
to fix the prices in such a way that software failure rate is not greater than that come out to be at cut of point.

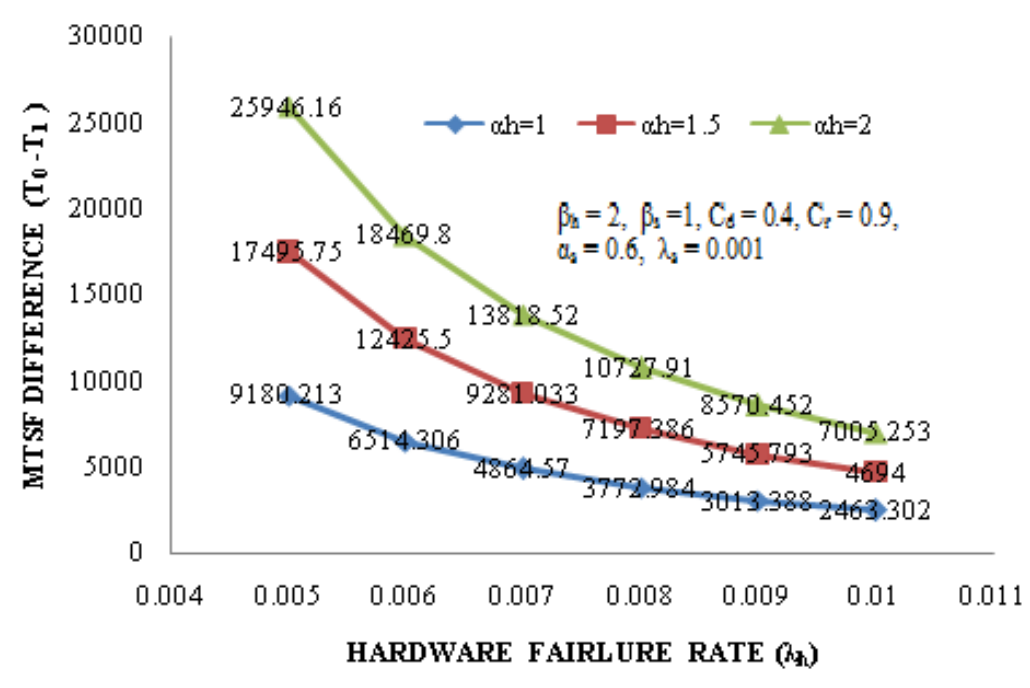

Fig. 4. Mean Time to System Failure versus hardware failure rate for different values of hardware repair rate

In the fig. 4, difference of mean times to system failure ( $\left.\mathbf{T}_{\mathbf{0 1}}-\mathbf{T}_{\mathbf{0 2}}\right)$ decreases as hardware failure rate $\left(\lambda_{h}\right)$ increases and has higher values for the higher values of hardware repair rate $\left(\alpha_{\mathrm{h}}\right)$. It can also be concluded from the graph that mean time to system failure $\left(\mathrm{T}_{01}\right)$ of the model $\mathrm{I}$ is greater than the mean time to system failure $\left(\mathrm{T}_{02}\right)$ of the model II.

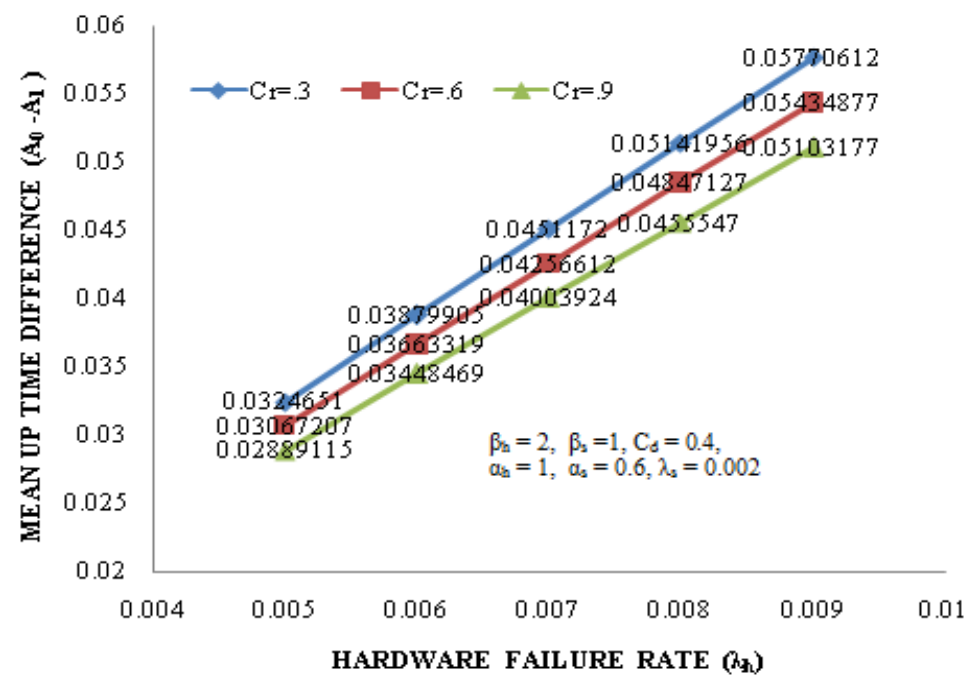

Fig. 5. Mean Up Time versus hardware failure rate for different values of fault recovery coverage 
Fig. 5 depicts the behaviour of the difference of mean up times (A01-A02) of the model I and model II with respect to the hardware failure rate $\left(\lambda_{h}\right)$ for different values of fault recovery coverage $\left(c_{r}\right)$. It can be observed from the graph that difference of mean up times ( $\left.\mathbf{A}_{01}-\mathbf{A}_{02}\right)$ of the system increases as hardware failure rate $\left(\lambda_{\mathrm{h}}\right)$ increases and has lower values for the higher values of fault recovery coverage $\left(c_{r}\right)$. So it can be concluded that the values of mean up time $\left(\mathrm{A}_{02}\right)$ of model II are greater than the values of mean up time $\left(\mathrm{A}_{01}\right)$ of model given in model $\mathrm{I}$.



Fig.6. Mean Up Time versus software failure rate for different values of fault detection coverage

In the fig. 6, difference of mean up time increases with increase in the values of software failure rate and have higher values for higher values of fault detection coverage. Thus, from the graph, we conclude that model II is better than model I. 


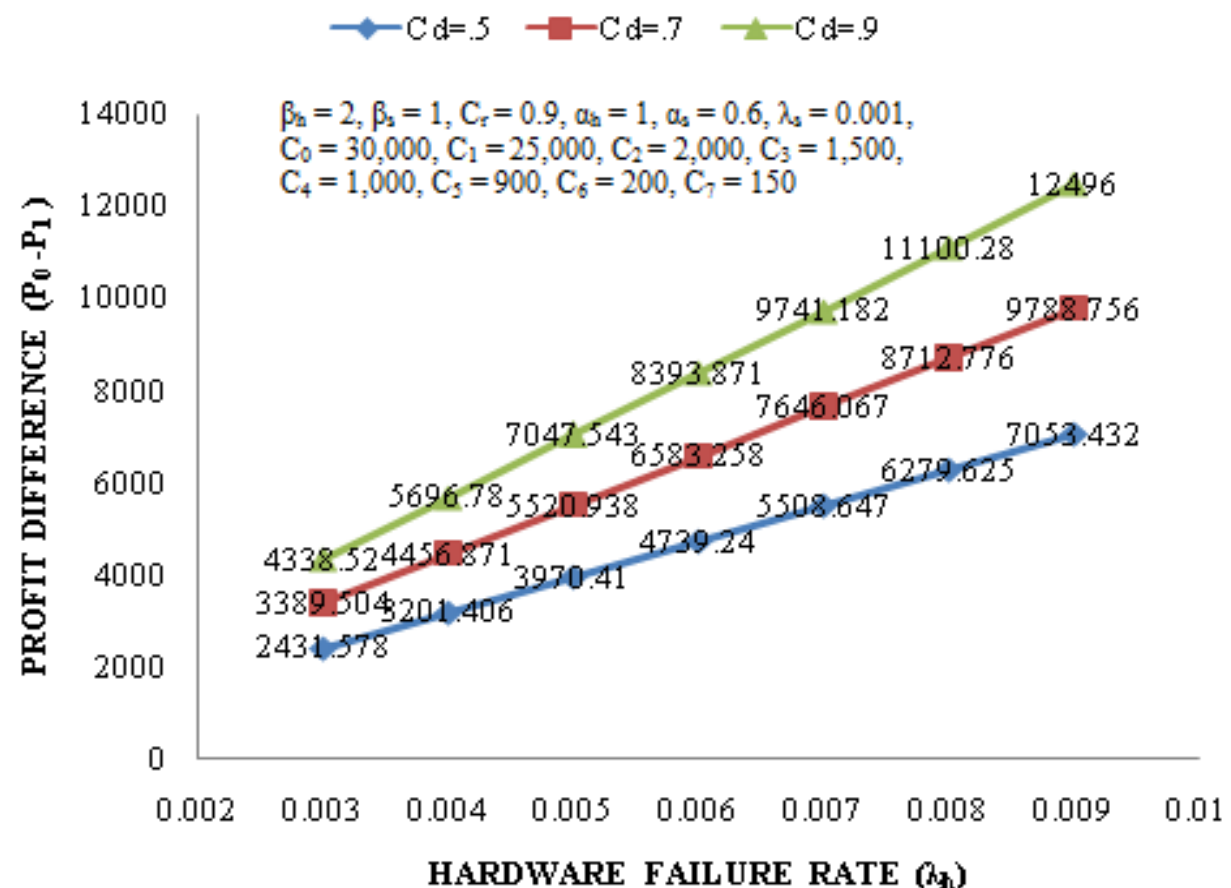

Fig. 7. Profit versus hardware failure rate for different values of fault detection coverage

In the fig. 7 profit difference $\left(\mathbf{P}_{01}-\mathbf{P}_{02}\right)$ of the system increases with increase in the values of hardware failure rate and have higher values for higher values of fault detection coverage. It can be concluded that the value of profit $\left(\mathrm{P}_{02}\right)$ of model II is greater than the values of profit $\left(\mathrm{P}_{01}\right)$ of model $\mathrm{I}$.

\section{CONCLUSION}

Comparing the models, we reached on conclusion that we have obtained different measures of the system effectiveness by taking the assumed values of hardware/software failure rate(s), fault detection coverage, fault recovery coverage and hardware/software repair rate(s). Overall, Model II is better than model I. The models discussed here can be fitted by the users of such systems. The users of such systems, while fitting the models discussed, can take the real values and can obtain various cut-off points of the desired rates, costs, revenue, etc. proceeding in the similar fashion as we have done in the paper. By doing so, they can get very interesting and useful results which may help them in attaining wonderful momentary gains. 


\section{REFERENCES}

[1] Amari, S.V., Duhan, J.B. and Misar, R.B., 1999. Optimal Reliability of System Subject to Imperfect Fault-Coverage, IEEE Transactions on Reliability, Vol.48, No. 3, pp. 275-284. DOI: 10.1109/24.799899.

[2] Boyd, M.A. and Monahan, C. M., 1995. Developing Integrated Hardware -Software System Reliability Models: Difficulties and Issues [For Digital Avionics]. Proceeding of the Digital Avionics Systems Conference, $14^{\text {th }}$ DASC, pp.193-198, Cambridge, USA. DOI: 10.1109/DASC.1995.482829.

[3] Friedman, M.A. and Tran, P., 1992. Reliability Techniques for Combined Hardware/Software Systems, Proceeding Annual Reliability and Maintainability Symposium, pp.290-293. http://www.dtic.mil/dtic/tr/fulltext/ u2/a256347.pdf

[4] Goel, L.R., Gupta, R. and Gupta, P., 1983. Analysis of a Two-Unit Hot Standby System with Three Modes, Microelectronics Reliability, Vol. 23(6), pp 1029-1033. DOI: 10.1016/0026-2714(83)90515-2.

[5] Iyer, R.K., 1985. Hardware Related Software Errors: Measurement and Analysis. IEEE Transactions on Software Engineering, Vol.11, pp.223-230. DOI:10.1109/TSE.1985.232198.

[6] Kanoun, K. and Ortalo-Borrel, M., 2000. Fault-Tolerant System Dependency -Explicit Modeling of Hardware and Software Component-Interactions, IEEE Trans. Reliability, Vol. 49, No. 4, pp. 363-376. DOI: 10.1109/24.922489.

[7] Kumar, M. and Kumar, R., 2016. Comparative Availability and Profit Analysis of Stochastic Models on Hardware-Software System, Journal of Mathematics \& System Sciences, Vol.12, No.-1-2, pp.1-10. ISSN: 2159-5291.

[8] Kumar, R. and Kumari, S., 2013. Analysis of a Stochastic Model on a TwoUnit Hot Standby Combined Hardware-Software System, International Journal of Computer Applications, Volume 78, No.2, pp. 29-35. DOI: 10.1.1.402.6974.

[9] Parashar, B. and Bhardwaj, N., 2013. A Comparative Study of Profit Analysis of Two Reliability Models on a Two-Unit PLC System, International Journal of Scientific and Engineering Research, Vol. 4, Issue 4, pp. 400-409.

[10] Rizwan, S.M., Khurana, V., and Taneja, G., 2005. Reliability Modelling of a Hot Standby PLC System, International Conference on Communication, Computer \& Power, pp. 486-489.

[11] Sharma and Kaur, 2016. Comparative Study of Two Standby Systems with Concept of Priority to Failed Unit, International Journal of Science, Engineering and Technology Research, Vol. 5(4), pp. 1202-1206. 
[12] Teng, X., Pham, H. and Jeske, D.R., 2006. Reliability Modeling of Hardware and Software Interactions and its Applications, IEEE Transaction on Reliability, Vol.55, pp.571-577. DOI: 10.1109/TR.2006.884589.

[13] Trivedi, K.S., Kim, D.S. and Ghosh, R., 2013. System Availability Assessment using Stochastic Models, International Journal of Applied Mathematical Research, Vol.29, pp. 112-113.

[14] Welke, S.R., Johnson, B.W. and Aylor, J.H., 1995. Reliability Modeling of Hardware/Software Systems, IEEE Transaction on Reliability, Vol.44, pp.413-418. DOI: $10.1109 / 24.406575$.

\section{AUTHORS CONTRIBUTIONS:}

The literature pertaining to hardware-software systems have been studied through various research papers and books, investigated the problem, designed the stochastic model and calculated various indices for the systems performance. Comparison and graphical analyses are carried out and important conclusions regarding reliability, availability and profit incurred to the system are drawn.

\section{Conflict of Interest:}

There is no conflict of interest regarding the publication of this manuscript. 
